\title{
Avaliação do desenvolvimento físico de lactentes no contexto da atenção primária à saúde
}

\section{Evaluation of the physical development of infants in the context of primary health care}

\author{
Pedro Raul Saraiva Rabelo' •Wesley Soares de Melo $^{2} \bullet$ Samara Pereira Souza Mariano ${ }^{3}$ \\ Maria do Socorro Távora de Aquino ${ }^{4}$ Flávia Paula Magalhães Monteiro ${ }^{5}$
}

\begin{abstract}
RESUMO
Objetivo: avaliar o desenvolvimento físico de lactentes no contexto do nível primário de atenção à saúde. Método: estudo transversal, descritivo e quantitativo, realizado em unidades de atenção primária à saúde de três cidades da região do Maciço de Baturité- CE:Acarape, Redenção e Itapiúna.A amostra foi constituída por lactentes acompanhados em unidades de saúde de acordo com a demanda apresentada.A coleta ocorreu entre março a abril de 2018 mediante dados sobre a caracterização geral e antropométrica do lactente, avaliação do desenvolvimento motor, com base no teste de desenvolvimento de Denver Il e análise de reflexos primitivos. Os dados foram agrupados em planilhas Excel e compilados no software SPSS versão 20.0. Resultados: Participaram do estudo 75 crianças na fase de lactente. Um percentual de $60 \%$ dos lactentes era do sexo masculino, com média de 7,7 meses de idade. Na avaliação antropométrica dos escores z, a relação IMC/idade apresentou maior percentual de inadequação $(66,7 \%)$, apresentando-se, na maioria, como acima do esperado. Na avaliação do desenvolvimento motor, houve risco de atraso em $13,3 \%$ dos lactentes ( $4 \%$ no motor fino e $9,3 \%$ no motor grosso). Na avaliação dos reflexos, em grande parte dos lactentes $(85,3 \%)$ estavam presentes de acordo com o esperado para a idade Conclusões: nota-se um perfil de crianças com peso acima do adequado. Poucas alterações no desenvolvimento motor e nos reflexos foram constatadas, apontando para uma possível e adequada estimulação dos responsáveis e correta orientação dos profissionais de saúde. Palavras-chave: Crescimento e Desenvolvimento; Desenvolvimento Infantil; Lactente; Enfermagem pediátrica;Atenção Primária à Saúde.
\end{abstract}

\begin{abstract}
Objective:To evaluate the physical development of infants in the context of primary health care level. Method: a cross-sectional, descriptive and quantitative study, carried out in primary health care units in three cities in the Massif de Baturité-CE region:Acarape, Redenção and Itapiúna. The sample consisted of infants followed in health units according to the demand presented.The collection took place between March and April 2018 through data on the general and anthropometric characterization of the infant, assessment of motor development, based on the Denver II development test and analysis of primitive reflexes. Data were grouped in Excel spreadsheets and compiled using SPSS version 20.0 software. Results: Seventy-five infants participated in the study.A percentage of $60 \%$ of the infants were male, with an average of 7.7 months of age. In the anthropometric evaluation of the z-scores, the BMI / age ratio presented a higher percentage of inadequacy $(66.7 \%)$, and was mostly higher than expected. In the assessment of motor development, there was a risk of delay in $13.3 \%$ of infants ( $4 \%$ in fine engine and $9.3 \%$ in coarse engine). In the evaluation of reflexes, most of the infants $(85.3 \%$ ) were present as expected for their age. Conclusions: a profile of children with overweight is noted. Few changes in motor development and reflexes were found, pointing to a possible and adequate stimulation of those responsible and correct guidance of health professionals. Keywords: Growth and Development; Child Development; Infant; Pediatric Nursing; Primary Health Care.
\end{abstract}

NOTA

I Enfermeiro. Universidade da Integração Internacional da Lusofonia Afro-Brasileira. E-mail: raulrabelo7@gmail.com

2 Mestre em Enfermagem. Universidade da Integração Internacional da Lusofonia Afro-Brasileira, Departamento de Enfermagem. Endereço: Rodovia CE 060, Km5I,Acarape-Ce, Brasil.E-mail:wesley_16I@hotmail.com

3 Mestre em Enfermagem. Universidade da Integração Internacional da Lusofonia Afro-Brasileira. E-mail: samarapereiradesouza@gmail.com

4 Mestranda em Enfermagem pela Universidade da Integração Internacional da Lusofonia Afro-Brasileira. E-mail: socorrotavora I@hotmail.com

5 Doutora em Enfermagem. Docente do curso de Graduação em Enfermagem e do Programa de Pós-Graduação em Enfermagem da Universidade da Integração Internacional da Lusofonia Afro-Brasileira.E-mail: flaviapmm@unilab.edu.br 


\section{INTRODUÇÃO}

O desenvolvimento infantil é um fenômeno amplo, contínuo e dinâmico que engloba inúmeros fatores e se constrói a partir das interações criança-criança, criançafamília e criança- ambiente. Desse modo, o desenvolvimento da criança se baseia em fatores genéticos, no crescimento e nas relações com os ambientes físico, social e cultural. Pode ser, ainda, dividido em vários domínios, dentre os quais, podem ser destacados: o psicossocial, o cognitivo e o físico ${ }^{(1,2)}$.

Dessa forma, o desenvolvimento da criança compreende algumas etapas/fases designadas de acordo com o ciclo vital da criança e sua idade. No Brasil, conforme as faixas etárias consideradas pelo Ministério da Saúde (MS), a fase de lactente compreende a idade de I até 24 meses de idade, que é o período em que se preconiza a duração do aleitamento materno, assim como também é a definição adotada no Descritores em Ciências da Saúde (DeCS) ${ }^{(3-5)}$.

O desenvolvimento físico, por si só, também é um fenômeno denso e vasto, englobando o crescimento, os reflexos primitivos, as habilidades motoras, dentre outros diversos fatores. O crescimento, por sua vez, pode ser definido como o aumento físico do corpo de forma global ou de uma parte específica. Os processos de hipertrofia (aumento do tamanho das células) e de hiperplasia (aumento no número das células) é que determinam esse fenômeno. Assim, o crescimento pode ser mensurado por meio da antropometria, utilizando medidas como peso e comprimento, principalmente; além de circunferências braquial e cefálica, bem como as pregas cutâneas tricipital e subescapular ${ }^{(1,2)}$.

Os reflexos primitivos são reações não intencionais do indivíduo que auxiliam na avaliação da integridade física e neurológica da criança, visto que, existem períodos específicos de início e término para cada um desses reflexos. Desse modo, a ausência deles, no período apropriado, podem indicar alterações importantes no processo de saúde e desenvolvimento do lactente ${ }^{(6,7)}$.

Além do crescimento e dos reflexos primitivos, o domínio físico engloba, ainda, as Habilidades Motoras Grossas (HMG) e Habilidades Motoras Finas (HMF). As HMG são as que se relacionam à utilização dos músculos maiores que permitem movimentos corporais tais como andar, correr e pular.Ao passo que as HMF utilizam músculos menores, que se relacionam com a coordenação entre olhos e mãos, que é evidenciada pelo movimento de pegar em "pinça". Vale destacar que, as maiores e mais importantes evoluções inerentes ao desenvolvimento motor, ocorrem na fase de lactância ${ }^{(2,8,9)}$.

Com base nisso, torna-se fundamental o acompanhamento do desenvolvimento físico de lactentes, visto que verificar o quanto o organismo cresce e desenvolve ha- bilidades motoras de forma adequada ou não permite articular estratégias interventivas mais eficientes para melhoria dos resultados da criança e, consequentemente, evitar ou amenizar problemas futuros no desenvolvimento que possam vir a repercutir na vida adulta ${ }^{(10)}$.

Nessa perspectiva, durante a consulta de puericultura, uma das atividades mais importantes que o enfermeiro realiza é o acompanhamento do desenvolvimento físico. No entanto, diante de tantos fatores que compõem este fenômeno, algumas fragilidades são observadas nesta consulta. Desse modo, percebe-se que o acompanhamento do crescimento ocorre por meio das verificação das medidas antropométricas, o que já é consolidado e tem como base os gráficos da caderneta de saúde da criança, conforme recomenda o Ministério da Saúde do Brasil. Os reflexos primitivos também são amplamente observados durante a consulta. $O$ desenvolvimento motor, no entanto, muitas vezes não consegue ser avaliado de forma tão concreta, pois os marcos no desenvolvimento abordados na caderneta, apresentam apenas pontos específicos em faixas etárias amplas ${ }^{(11)}$.

Nesse sentido, os profissionais de saúde da atenção primária abordam a avaliação do desenvolvimento de forma geral. Entretanto, analisar o domínio físico, em separado, permite o aprofundamento do enfermeiro acerca das peculiaridades do fenômeno, possibilitando a identificação de possíveis atrasos e, assim, a definição de intervenções mais eficientes. $O$ problema que dificulta essa avaliação é o desconhecimento dos itens que devem ser avaliados pelos profissionais, bem como, a falta de um instrumento padronizado que avalie de forma mais completa o desenvolvimento motor. Nesse contexto, existem inúmeros testes e escalas de avaliação já padronizados, validados e utilizados amplamente em todo mundo. Entretanto, um teste que vem sendo bastante empregado no Brasil, embora tenha poucos estudos de validação, é o Teste de Triagem do Desenvolvimento de Denver II (TTDD-II). Ele avalia, separadamente, os desenvolvimentos pessoalsocial, da linguagem e, com relação ao desenvolvimento físico, analisa os domínios motores fino e grosseiro ${ }^{(12)}$.

O teste pode ser aplicado por vários profissionais da saúde, sendo simples e de fácil manejo, o que aumenta seu espectro de possibilidade de utilização, podendo ser utilizado em diversos locais, inclusive, em Unidades de Atenção Primária à Saúde (UAPS). Embora não tenha alto valor prognóstico, é um excelente teste de comparação entre a criança analisada e o restante da população na faixa etária, possibilitando a observação de marcos no desenvolvimento. Ou seja, permite avaliar a condição atual do desenvolvimento maturacional da criança, mas não é recomendado ser utilizado como um instrumento diagnóstico ${ }^{(13,14,15)}$. Assim, tem-se a seguinte questão norteadora: a utilização do TTDD-II, em conjunto com 
os parâmetros de acompanhamento do crescimento e testes de reflexos pode contribuir para que o desenvolvimento físico seja melhor avaliado no contexto da atenção primária?

Nessa perspectiva, o estudo contribui para que o desenvolvimento físico possa ser acompanhado de maneira ainda mais completa. Proporcionando uma expansão da visualização da forma de avaliação deste domínio do desenvolvimento infantil, com instrumentos que abordem de forma lúdica e eficiente todos os componentes do fenômeno e, por conseguinte, auxiliando na melhoria da atuação do enfermeiro no que se refere à intervenção precoce de possíveis alterações. Dessa maneira, o objetivo do estudo consiste em avaliar o desenvolvimento físico de lactentes no contexto do nível primário de atenção à saúde.

\section{MÉTODO}

Estudo transversal e descritivo, com abordagem quantitativa, realizado em três cidades da região do maciço de Baturité-CE (Itapiúna, Redenção e Acarape). A população do estudo foi crianças na fase de lactentes atendidos nas UAPS da zona urbana dos municípios da região supracitada (I UAPS por município). A amostra obtida foi do tipo não-probabilística por conveniência de acordo com a demanda das unidades do estudo, totalizando 75 crianças (em média, em cada UAPS eram atendidas 25 crianças, mensalmente). Nesse sentido, o recrutamento dos responsáveis e lactentes aconteceu antes da consulta de puericultura, enquanto aguardavam atendimento, os quais eram convidados e, após o aceite em participar do estudo, eram encaminhados a uma sala que estivesse reservada nas próprias unidades de saúde. A entrevista e os testes foram realizados por dois acadêmicos de enfermagem simultaneamente, previamente treinados.

Desse modo, os critérios de inclusão foram: criança ser atendida em alguma das instituições, lócus do estudo e encontrar-se na faixa etária entre I mês a 2 anos de idade (lactentes). Enquanto isso, o critério de exclusão utilizado foi a criança não ter completado toda a avaliação.

A coleta ocorreu entre março e abril de 2018. Os instrumentos utilizados abordavam variáveis relacionadas ao lactente e à família, sendo divididos da seguinte maneira: (I) Perfil pessoal, sociodemográfico e clínico dos responsáveis pelos cuidados com o lactente; (2) Caracterização pessoal, clínica e alimentar dos lactentes; (3) Informações gestacionais e obstétricas maternas e neonatais; (4) Caracterização e avaliação dos escores z dos dados antropométricos dos lactentes; (5) Avaliação do desenvolvimento motor (DM) dos lactentes, com base no TTDD-II; e (6) Análise dos reflexos primitivos dos lactente, considerando os reflexos de Babinski, preensão palmar e plantar, de Moro, da marcha e o da sucção.
Nesse contexto, para a obtenção dos dados antropométricos foram utilizados equipamentos previamente testados, tais como: uma balança de mesa digital infantil, apropriada para verificação do peso de crianças na fase de lactente; um antropômetro científico; e uma fita métrica graduada em centímetros ${ }^{(2)}$. Já os escores $z$ peso/ idade (P/I), estatura/idade (E/I), IMC/idade (IMC/I) e perímetro cefálico/idade (PC/I) foram analisados conforme os gráficos das cadernetas de saúde da criança do Ministério da Saúde ${ }^{(I I)}$.

Com relação aos escores $z$, a classificação adotada do P/l foi: peso elevado para idade $(>+2)$, peso adequado para idade $(\geq-2$ e $\leq+2)$, baixo peso para idade $(\geq-3$ e $<$ $-2)$, muito baixo peso para idade $(<-3)$. Para classificação da E/I: estatura adequada para idade $(\geq-2)$, baixa estatura para idade $(\geq-3$ e $<-2)$, muito baixa estatura para idade $(<-3)$. No escore $Z$ na relação IMC/I, a classificação considerada foi: obesidade $(>+3)$, sobrepeso $(\leq+3$ e $>$ $+2)$, risco de sobrepeso $(\leq+2$ e $>+1)$, adequado $(\leq+$ I e $\geq-2$ ), magreza (<-2 e $\geq-3$ ) e magreza acentuada (< -3). A classificação utilizada para a relação PC/I foi: acima do esperado $(>+2)$, adequado $(\leq+2$ e $\geq-2)$ e abaixo do esperado $(<-2)$.

Para a avaliação do desenvolvimento motor foi realizada uma adaptação do TTDD-II, considerando apenas os itens de teste que se enquadravam na avaliação do desenvolvimento físico dos lactentes. Desse modo, do domínio do Desenvolvimento Motor Fino (DMF), que possuía 29 itens no total, foram considerados apenas 19 (do $1^{\circ}$ ao $19^{\circ}$ ) itens. Já para o Desenvolvimento Motor Grosso (DMG), de 32 itens, foram considerados apenas 21 (do $3^{\circ}$ a $23^{\circ}$ ) itens. Assim, alguns materiais foram necessários para a realização dos testes, tais como: pom -pom vermelho, chocalho, uvas passas, 6 cubos/blocos de empilhar, recipiente com diâmetro maior que os cubos/ blocos, folhas de papel sem pauta, lápis de cor ou grafite e brinquedos variados (bola, carro e bonecos/as) ${ }^{(12)}$.

Com relação a utilização do TTDD-II, foram seguidas as etapas:

$I^{\circ}$ ) etapa:Avaliação de cada item presente nos domínios Motor Grosso e Motor Fino

$\mathrm{Na}$ avaliação dos itens de cada domínio, foram executadas atividades para realização dos testes, sendo consideradas as seguintes denominações: Passou (quando a criança teve a resposta esperada para o teste); Falhou (quando não realizou o teste, conforme a resposta esperada) e; Sem oportunidade (quando por algum motivo o teste não pode ser realizado).

\section{$2^{\circ}$ ) etapa: Estabelecimento do resultado}

As denominações dadas aos itens na etapa anterior foram analisadas em conjunto: idade do lactente e percentil $(25 \%, 75 \%, 90 \%)$ de realização de cada atividade para a faixa etária da criança. Neste primeiro momento, 
essa denominação subsidiou o resultado da avaliação dos testes para cada item por meio da classificação:

Normal - quando a criança executou a atividade prevista para a idade ou não executou uma atividade realizada por menos de $75 \%$ das crianças da mesma idade;

Cuidado (atenção)- quando a criança não executou ou se recusou a realizar atividade que já é feita por 75 a $90 \%$ das crianças daquela idade;

Atraso - quando a criança não executou ou se recusou a realizar atividade que já é executada por mais de $90 \%$ das crianças que têm sua idade.

$3^{\circ}$ ) etapa: Classificação geral do domínio a partir da avaliação dos itens segundo a escala de Denver II:

Foram revisados os resultados de todos os itens de cada domínio (motor fino e motor grosso) para que fosse possível estabeler a classificação geral dos mesmos. A classificação final se deu deste modo:

Normal - a criança foi classificada como normal em todos os itens de teste ou apresenta uma classificação de "cuidado (atenção)" descrito anteriormente;

Risco de atraso - a criança apresentou duas ou mais classificações de "cuidado" e/ou pelo menos uma classificação de atraso;

Não testável - a criança se recusou a realizar mais de $50 \%$ dos testes para a sua faixa etária ${ }^{(16)}$.

No que se refere à avaliação dos reflexos primitivos, os resultados foram agrupados de acordo com as possibilidades de presença ou ausência do reflexo testado. Desse modo, foi classificado como presente ou ausente e, ainda quando o reflexo não era referente à idade em que o lactente se encontrava ou não era possível realizar o teste, foi classificado como não testado.

Todos os dados foram compilados no software Excel 2013, em planilhas, e a análise estatística descritiva foi realizada no programa Statistical Package for the Social Sciences (SPSS) versão 20.0, calculando-se média, desvio padrão, valores mínimo e máximo, frequência e porcentagem. Após isso, os dados foram apresentados em tabelas e discutidos com a literatura pertinente.

Ademais, todos os aspectos éticos foram respeitados conforme a Resolução 466/12(17), como também pela aprovação da pesquisa pelo Comitê de Ética em Pesquisa da Universidade Internacional da Lusofonia Afro-Brasileira (UNILAB), sob o número do parecer: 2.69l.7।4.

\section{RESULTADOS}

A maioria dos lactentes (60\%) era do sexo masculino, sendo que em todas os municípios houve um predomínio de meninos. Considerando o sexo das crianças, o município de Acarape-Ce apresentou $72 \%$ dos participantes meninos. A idade média geral foi de 7,7 meses, tendo os lactentes do município de Itapiúna-Ce um perfil mais jovem, com média de 5,9 meses, em relação ao mu- nicípio de Acarape-Ce (8,4 meses) e Redenção-Ce (8,9 meses). A maioria $(57,3 \%)$ das crianças tinham idade inferior a seis meses. Com relação à naturalidade, houve distribuição dos nascimentos, principalmente, entre os municípios do Estado do Ceará: Redenção (30,7\%), Baturité (24\%) e Fortaleza (20\%).

Quanto à imunização, 90,7\% dos lactentes estavam com o calendário vacinal atualizado. Dois (8\%) lactentes no município de Acarape-Ce tinham patologias congênitas relacionadas à deformidade óssea, ao passo que, no município de Itapiúna-Ce, também dois (8\%), tinham doenças congênitas (permanência da abertura do forame oval do coração e paralisia cerebral). Em Redenção-Ce não haviam casos de problemas de saúde congênitos. Em termos gerais, apenas três (4\%) de todos os lactentes apresentavam outro tipo de doença, a saber: asma, constipação e hérnia umbilical.

$\mathrm{Na}$ caracterização alimentar dos lactentes, pode-se destacar que 93,3\% dos lactentes não possuíam quaisquer tipo de alergias alimentares. Apenas um lactente (I,3\%), de Redenção-Ce, não pode ser amamentado por motivos adversos. Com relação ao aleitamento materno (AM), $82,7 \%$ dos lactentes ainda mamavam e, 32\% dos lactentes estavam em Aleitamento Materno Exclusivo (AME). No geral, $31,4 \%$ estiveram em AME por período menor que I mês, 35,3\% por período entre I e 4 meses, $29,4 \%$ por período de 4 a 6 meses, e apenas 3,9\% deles com duração maior que 6 meses.

Com relação ao perfil gestacional das mães dos lactentes, apenas $40 \%$ das gestações foram planejadas. No que se refere à avaliação ponderal das mães no período gestacional, $72 \%$ das mulheres estavam com o peso adequado durante a gravidez. No entanto, um percentual importante $(25,3 \%)$ estava com sobrepeso. Em $29,3 \%$ dos casos, houve alguma intercorrência gestacional, com destaque especial para a pré-eclâmpsia e Infecções no Trato Urinário (ITU). Todas as mães realizaram acompanhamento pré-natal, tendo feito, em média, cerca de oito consultas.

No que se refere aos dados obstétricos, a média de fiIhos entre as mães dos lactentes avaliados foi de, aproximadamente, duas crianças. $A$ idade materna no parto do lactente foi, em média, 25,4 anos $( \pm 5,9)$, variando entre 17 e 40 anos. A Idade Gestacional (IG) apresentada no parto foi, em média geral, de 39,6 semanas $( \pm 2,0)$.

Concernente às medidas antropométricas da criança ao nascer, o peso médio dos lactentes foi de 3260,2 gramas $( \pm 592,0)$, variando entre 1200 a 4800 gramas. Já o comprimento médio ao nascer foi de $49, \mathrm{Icm}( \pm 2,9)$, indo de $4 \mathrm{I}$ até $59 \mathrm{~cm}$.

Em relação às variáveis sociodemográficas das famílias dos lactentes, todos os cuidadores eram do sexo feminino, representados pelas mães, avós e tias.A média de idade foi $26,4( \pm 6,6)$ anos. Referente à escolaridade das cuidadoras, 
a média geral encontrada foi de $9,6( \pm 3, I)$ anos estudados. Nessa perspectiva, $50,6 \%$ de toda a amostra não havia concluído o ensino médio, sendo $4 \%$, ainda, analfabetos. Já no que concerne à ocupação, $69,3 \%$ estudava e/ou trabaIhava fora de casa, 30,7\% era dona de casa. Com relação ao estado civil, a maior parte ( $81,3 \%$ no geral) vivia com companheiro, sendo casada ou em união consensual.

No que se refere ao perfil clínico e hábitos de vida das cuidadoras, $48 \%$ das responsáveis tinham no momento ou já haviam tido algum tipo de vício, sendo o alcoolismo o mais amplamente citado ( $36 \%$ no geral), seguido do tabagismo (10,7\%) e uma mulher já havia sido usuária de drogas ilícitas. Com relação às condições de saúde, duas $(2,7 \%)$ tinham problemas de cunho psicológico/ mental, depressão e retardo mental. No geral, as cuidadoras apresentaram problemas de saúde relacionados ao sistema cardiovascular, trato respiratório e alergias.

Concernente às condições de moradia das famílias, todas as casas eram de alvenaria e $70 \%$ delas eram próprias. Levando em conta a situação econômica, a média mensal de renda familiar era de 794,5 reais $( \pm 7 \mid 8,2)$. Dessa maneira, considerando o Salário Mínimo (SM) vigente no Brasil à época do estudo $(R \$ 954,00)^{*}, 80 \%$ das famílias, no geral, possuíam renda mensal igual ou inferior a I salário mínimo; I3,3\%, por sua vez, apresentaram renda entre I SM até 2 SM; enquanto o restante $(6,7 \%)$ tinha renda superior a $2 \mathrm{SM}$.

A seguir, a tabela I apresenta a caracterização antropométrica e avaliação dos escores $\mathrm{z}$ dos lactentes em estudo.

Referente ao Índice de Massa Corpórea (IMC), a média geral foi de $19,0 \mathrm{~kg} / \mathrm{m} 2( \pm 2,3)$. A menor média foi do lactente do município de Acarape-Ce (I8,2kg/m2), já as médias de lactentes oriundos dos municípios de RedençãoCe e Itapiúna-Ce foram muito próximas, sendo $19,5 \mathrm{~kg} /$ $\mathrm{m} 2$ e $19,4 \mathrm{~kg} / \mathrm{m} 2$, respectivamente. Além disso, das cinco crianças (6,7\% do geral), que estavam obesas, quatro eram oriundas de Redenção-Ce (16\% da amostra da cidade).

Considerando os escores z de Perímetro Cefálico (PC)/idade, a única criança que apresentou PC abaixo do esperado (I,3\% do geral) foi do município de RedençãoCe. Dos seis lactentes ( $8 \%$ do geral) que apresentaram PC acima do esperado, a maioria foi de Redenção-Ce, que apresentou quatro casos ( $16 \%$ da amostra da cidade). Nesse aspecto, a média geral encontrada para o PC foi de $43, \mathrm{Icm}( \pm 3,4)$, sendo a média de lactentes do município de Itapiúna-Ce a mais discrepante com relação

TABELA 1 - Caracterização antropométrica e avaliação dos escores $z$ dos lactentes, conforme a distribuição de cada cidade. Redenção-CE, Brasil, 2018.

\begin{tabular}{|c|c|c|c|c|c|c|c|c|}
\hline \multirow{2}{*}{ Medidas antropométricas } & \multicolumn{2}{|c|}{ Acarape $(n=25)$} & \multicolumn{2}{|c|}{ Redenção (n=25) } & \multicolumn{2}{|c|}{ Itapiúna $(n=25)$} & \multicolumn{2}{|c|}{ Geral $(n=75)$} \\
\hline & Média & {$\left[\mathrm{DP}^{1}\right]$} & Média & [DP] & Média & [DP] & Média & [DP] \\
\hline Peso (g) & 8616,8 & {$[2679,4]$} & 9113,0 & {$[1961,2]$} & 7540,8 & {$[2707,3]$} & 8537,1 & {$[2344,4]$} \\
\hline Comprimento $(\mathrm{cm})$ & 68,1 & {$[10,2]$} & 68,3 & {$[7,9]$} & 62,9 & {$[8,1]$} & 66,4 & {$[9,0]$} \\
\hline $\mathrm{IMC}^{2}$ & 18,2 & {$[2,2]$} & 19,5 & {$[2,9]$} & 19,4 & {$[1,7]$} & 19,0 & {$[2,3]$} \\
\hline $\mathrm{PC}^{3}(\mathrm{~cm})$ & 43,3 & {$[4,0]$} & 43,6 & {$[3,3]$} & 42,2 & {$[2,8]$} & 43,1 & {$[3,4]$} \\
\hline Escores $z^{4}$ & $\mathbf{N}$ & $\%$ & $\mathbf{N}$ & $\%$ & $\mathbf{N}$ & $\%$ & $\mathbf{N}$ & $\%$ \\
\hline \multicolumn{9}{|l|}{ Peso/idade } \\
\hline Elevado & 00 & 0,0 & 03 & 12,0 & 03 & 12,0 & 06 & 8,0 \\
\hline Adequado & 25 & 100,0 & 21 & 84,0 & 22 & 88,0 & 68 & 90,7 \\
\hline Baixo & 00 & 0,0 & 01 & 4,0 & 00 & 0,0 & 01 & 1,3 \\
\hline \multicolumn{9}{|l|}{ Comprimento/idade } \\
\hline Elevado & 01 & 4,0 & 02 & 8,0 & 00 & 0,0 & 03 & 4,0 \\
\hline Adequado & 21 & 84,0 & 19 & 76,0 & 21 & 84,0 & 61 & 81,3 \\
\hline Baixo & 03 & 12,0 & 03 & 12,0 & 04 & 16,0 & 10 & 13,3 \\
\hline Muito baixo & 00 & 0,0 & 01 & 4,0 & 00 & 0,0 & 01 & 1,3 \\
\hline \multicolumn{9}{|l|}{ IMC²/idade } \\
\hline Obesidade & 00 & 0,0 & 04 & 16,0 & 01 & 4,0 & 05 & 6,7 \\
\hline Sobrepeso & 07 & 28,0 & 08 & 32,0 & 09 & 36,0 & 24 & 32,0 \\
\hline Risco de sobrepeso & 07 & 28,0 & 06 & 24,0 & 07 & 28,0 & 20 & 26,7 \\
\hline Adequado & 10 & 40,0 & 07 & 28,0 & 08 & 32,0 & 25 & 33,3 \\
\hline Magreza & 01 & 4,0 & 00 & 0,0 & 00 & 0,0 & 01 & 1,3 \\
\hline \multicolumn{9}{|l|}{$\mathrm{PC}^{3} /$ idade } \\
\hline Acima do esperado & 01 & 4,0 & 04 & 16,0 & 01 & 4,0 & 06 & 8,0 \\
\hline Adequado & 24 & 96,0 & 20 & 80,0 & 24 & 96,0 & 68 & 90,7 \\
\hline Abaixo do esperado & 00 & 0,0 & 01 & 4,0 & 00 & 0,0 & 01 & 1,3 \\
\hline
\end{tabular}

1DP: Desvio Padrão; 2IMC: Índice de Massa Corporal; 3PC: Perímetro Cefálico; 4Escores z analisados conforme os parâmetros das cadernetas de saúde da criança (BRASIL, 2015). *Fonte: pesquisa direta. 
ao lactente do município de Redenção-Ce e Acarape-CE, tendo média I, I cm e I,4cm inferior, respectivamente.

A tabela 2 trata sobre a avaliação dos domínios motores do desenvolvimento dos lactentes, tendo como base no TTDD-II, de acordo com a distribuição entre as cidades lócus do estudo.
No que se refere à avaliação do desenvolvimento motor com base no TTDD-II, no desenvolvimento motor fino (DMF), no que concerne ao resultado da avaliação dos itens, apenas $0,9 \%$ dos lactentes apresentou Atraso e I,9\% dos lactentes se enquadaram na avaliação de cuidado (atenção). Porém, na avaliação geral do Teste de

TABELA 2 - Avaliação do desenvolvimento motor fino (DMF) e grossO (DMG) dos lactentes, conforme a distribuição por cada cidade, com base no teste de triagem de desenvolvimento de Denver II (TTDD-II).

Redenção-CE, Brasil, 2018.

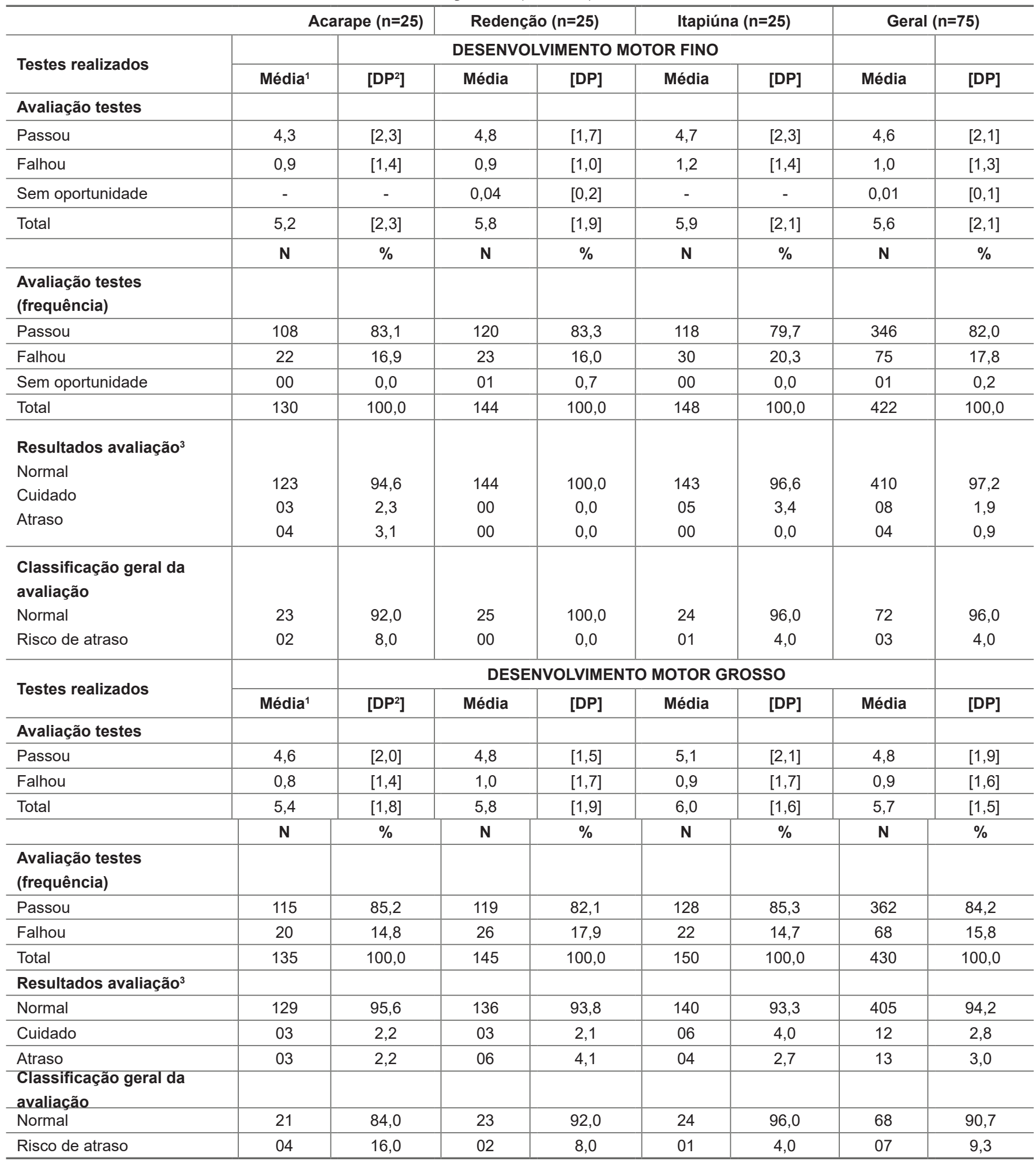

1 Média obtida pela divisão da quantidade de testes em que as crianças passaram ou falharam pelo número total de crianças; 2 Desvio Padrão; 3 De acordo com cada item testado. *Fonte: pesquisa direta. 
Denver II, $4 \%$ dos lactentes se encontravam em Risco para atraso para o desenvolvimento motor fino, enquanto $96 \%$ estava com a classificação Normal para o desenvolvimento físico.

Por outro lado, na avaliação do desenvolvimento motor grosso (DMG), no que concerne a cada item, apenas $3 \%$ dos lactentes apresentou Atraso e 2,8\% dos lactentes se enquadaram na avaliação de cuidado (atenção). Enquanto na avaliação geral do Teste de Denver II, sete $(9,3 \%)$ lactentes apresentaram Risco de atraso para o desenvolvimento físico, sendo os lactentes do município de Acarape com maior predomínio dos casos, o qual contabilizou quatro casos ( $16 \%$ da amostra da cidade).

A tabela 3, por sua vez, aborda sobre a análise feita com alguns dos principais reflexos primitivos que devem ser realizados em lactentes, conforme a sua faixa etária.

Em relação aos reflexos primitivos, todos os testes foram avaliados conforme a recomendação da faixa etária. O reflexo de Moro e da Marcha tiveram um maior percentual de não testados pois as crianças não se enquadravam na faixa de idade preconizada para a realização dos mesmos. Dos $4 \%$ de ausência do resultado esperado no reflexo de Babinski, uma das crianças já era diagnosticada com déficit neurológico, denominada paralisia cerebral.

Dessa forma, observa-se que houve um preodmínio de avaliações positivas entre os lactentes, conforme o teste empregado. Isso implica dizer que tais crianças apresentam adequadas condições de saúde no que se refere ao seu desenvolvimento físico, mesmo diante das limitações impostas pelo ambiente, cuidado e condições de saúde, tendo em vista que o desenvolvimento infantil é amplo, integrado e interrelacionado a outros domínios da esfera biológica do ser humano.

\section{DISCUSSÃO}

O estudo mostrou que a maior parte dos lactentes era do sexo masculino. Nesse aspecto, estudo de avaliação do desenvolvimento infantil, também com base no TTDD-II apontou predominância de crianças do sexo masculino ${ }^{(18)}$. A idade média geral foi de, aproximadamente, oito meses. Para isso, reforça-se que as consultas de puericultura são realizadas regularmente e perfazem um total de pelo menos sete no primeiro ano ${ }^{(11)}$. Por outro lado, por se tratar do acompanhamento da criança saudável, apresenta uma maior frequência de comparecimento nos primeiros meses de vida, pois é nesse período que as mães/responsáveis têm maiores dúvidas e preocupações quanto aos cuidados ${ }^{(19,20)}$.

Para compor a avaliação do desenvolvimento físico da criança, a investigação do perfil clínico do lactente é importante, visto que o desenvolvimento infantil é influen-

TABELA 3 - Avaliação dos reflexos primitivos dos lactentes, conforme a distribuição por cidades.

Redenção-CE, Brasil, 2018.

\begin{tabular}{|c|c|c|c|c|c|c|c|c|}
\hline \multirow{2}{*}{ Reflexos } & \multicolumn{2}{|c|}{ Acarape $(n=25)$} & \multicolumn{2}{|c|}{ Redenção (n=25) } & \multicolumn{2}{|c|}{ Itapiúna $(n=25)$} & \multicolumn{2}{|c|}{ Geral $(n=75)$} \\
\hline & $\mathbf{N}$ & $\%$ & $\mathbf{N}$ & $\%$ & $\mathbf{N}$ & $\%$ & $\mathbf{N}$ & $\%$ \\
\hline \multicolumn{9}{|l|}{ Babinsk } \\
\hline Presente & 24 & 96,0 & 24 & 96,0 & 24 & 96,0 & 72 & 96,0 \\
\hline Ausente & 01 & 4,0 & 01 & 4,0 & 01 & 4,0 & 03 & 4,0 \\
\hline \multicolumn{9}{|c|}{ Preensão palmar } \\
\hline Presente & 18 & 72,0 & 19 & 76,0 & 23 & 92,0 & 60 & 80,0 \\
\hline Ausente & 01 & 4,0 & 00 & 0,0 & 00 & 0,0 & 01 & 1,3 \\
\hline Não testado & 06 & 24,0 & 06 & 24,0 & 02 & 8,0 & 14 & 18,7 \\
\hline \multicolumn{9}{|c|}{ Preensão plantar } \\
\hline Presente & 17 & 68,0 & 19 & 76,0 & 22 & 88,0 & 58 & 77,3 \\
\hline Ausente & 00 & 0,0 & 00 & 0,0 & 01 & 4,0 & 01 & 1,3 \\
\hline Não testado & 08 & 32,0 & 06 & 24,0 & 02 & 8,0 & 16 & 21,3 \\
\hline \multicolumn{9}{|l|}{ Moro } \\
\hline Presente & 13 & 52,0 & 11 & 44,0 & 18 & 72,0 & 42 & 56,0 \\
\hline Ausente & 01 & 4,0 & 00 & 0,0 & 01 & 4,0 & 02 & 2,7 \\
\hline Não testado & 11 & 44,0 & 14 & 56,0 & 06 & 24,0 & 31 & 41,3 \\
\hline \multicolumn{9}{|l|}{ Marcha } \\
\hline Presente & 14 & 56,0 & 03 & 12,0 & 04 & 16,0 & 21 & 28,0 \\
\hline Ausente & 00 & 0,0 & 01 & 4,0 & 01 & 4,0 & 02 & 2,7 \\
\hline Não testado & 11 & 44,0 & 21 & 84,0 & 20 & 80,0 & 52 & 69,3 \\
\hline \multicolumn{9}{|l|}{ Sucção } \\
\hline Presente & 20 & 80,0 & 18 & 72,0 & 23 & 92,0 & 61 & 81,3 \\
\hline Ausente & 00 & 0,0 & 02 & 8,0 & 00 & 0,0 & 02 & 2,7 \\
\hline Não testado & 05 & 20,0 & 05 & 20,0 & 02 & 8,0 & 12 & 16,0 \\
\hline
\end{tabular}

*Fonte: pesquisa direta. 
ciado pelas condições de saúde geral apresentadas pela criança.Assim, mesmo que em pequena proporção, alguns lactentes apresentaram problemas de saúde que têm relação direta com atraso no desenvolvimento, a saber, problemas cardíacos, neurológicos, respiratórios e ósseos ${ }^{(21)}$. Desse modo, entende-se que qualquer alteração no processo de saúde do lactente tem repercussão negativa para o desenvolvimento, especialmente, quando crônicas ${ }^{(2)}$.

Por outro lado, a imunização é um importante modo preventivo para $\circ$ acometimento de doenças ${ }^{(22)}$. Assim, na amostra avaliada, notou-se adequação no calendário vacinal, configurando-se como um fator protetivo para o desenvolvimento. Resultado semelhante, com $87,97 \%$ de cumprimento do calendário de vacinas, foi registrado em estudo realizado com 108 crianças em uma unidade de atenção primária à saúde (UAPS) em Belém-PA(23).

Outro fator protetor importante para o crescimento e o desenvolvimento saudável é o aleitamento materno $(\mathrm{AM})^{(\mathrm{II})}$. E, no presente estudo, 82,7\% dos lactentes ainda mamavam. Um número bastante superior à média geral do Brasil nos últimos anos, visto que, em 2013, a prevalência de AM foi de apenas 52, 1\%(24). Em estudo realizado com 27 lactentes, em uma unidade hospitalar de Redenção - CE (uma das cidades lócus do estudo), 55,5\% dos lactentes já estavam desmamados ${ }^{(2)}$. Isso remete a uma melhoria na eficácia do incentivo ao $A M$ e, ainda, confirma a evidência de que o $A M$ ofertado de maneira inadequada pode levar a problemas de saúde passíveis de hospitalização. Já ocorrência da AME foi inferior, visto que nos municípios de Acarape e Redenção $50 \%$ dos lactentes estavam em AME. Ainda assim, os percentuais apresentados foram muito superiores quando comparadas ao cenário nacional que, em 2013 , foi de menos de $37 \%(24)$.

As informações gestacionais e obstétricas das mães dos lactentes também constituem enfoque sobre a avaliação do desenvolvimento físico ${ }^{(25)}$.Apesar disso, no presente estudo todas as mães realizaram pré- natal, o que é um fator protetivo, já que o acompanhamento da gestação permite a prevenção de complicações na gestante, as quais exercem ligação direta com riscos ou agravos ao feto com impacto negativo no seu desenvolvimento( ${ }^{(26)}$. No entanto, ainda houve uma incidência de $29,3 \%$ dessas complicações, sendo por causa evitáveis (pré-eclâmpsia e infecções no trato urinário), o que remete a uma provável necessidade da melhoria da assistência pré-natal nas cidades estudadas. Concernente à Idade Gestacional, $78,8 \%$ dos lactentes nasceu à termo, apresentando uma média de peso e comprimento ao nascer considerada dentro dos parâmetros normais, embora algumas crianças tenham apresentado peso bem inferior ${ }^{(27,28)}$.

Grande parte das cuidadoras tinham idade superior a 18 e inferior a 30 anos, sendo que, $81,3 \%$ vivia com companheiro, esses dados podem ser considerados proteti- vos para o desenvolvimento infantil. No entanto, 50,6\% não havia concluído o ensino médio, $69,3 \%$ estudava e/ ou trabalhava fora de casa e a renda familiar mensal era, em $80 \%$ dos casos, menor ou igual a I SM, constituindo fatores de risco. $O$ fato de estudar ou trabalhar fora de casa pode dificultar $\circ$ incentivo e o acompanhamento eficaz do desenvolvimento(29).

$\mathrm{Na}$ avaliação antropométrica peso/idade, encontrou-se que $90,7 \%$ das crianças estava dentro do intervalo normal segundo classificação recomendada pelo Ministério da Saúde. No entanto, vale destacar nesta pesquisa que houve um número maior de lactentes com peso elevado para idade do que baixo para idade, resultado diferente do encontrado na literatura. Em estudo realizado, no ano de 2000, no Brasil, não houve casos de P/l elevado, enquanto 5,4\% apresentavam peso baixo ${ }^{(30)}$. Da mesma forma, em 201 I, estudo realizado no Paraná, apresentou apenas casos de baixo peso $(2,3 \%)^{(31)}$. Em estudo mais recente, realizado com 26 crianças, no estado do Ceará, o número de lactentes com risco de peso elevado foi $7,6 \%$ superior ao de $\mathrm{P} / \mathrm{l}$ baixo ${ }^{(2)}$. Isso aponta para uma inversão que vem ocorrendo nos últimos anos acerca do ganho de peso de crianças, com a crescente industrialização de produtos e hábitos de vida das famílias, o predomínio passou a ser de peso elevado, em todas as faixas etárias, inclusive, crianças $^{(32)}$.

No comprimento médio, $81,3 \%$ estavam dentro do intervalo normal.Assim, I3,3\% das crianças estavam com E/l baixa e I,3\% muito baixa.

No que se refere ao IMC, a menor média foi apresentada pelos lactentes do município de Acarape-Ce. Reunindo todos os lactentes que obtiveram escores $z$ acima do adequado, chega-se a um percentual de mais de $65 \%$ dos lactentes. Apenas um lactente apresentou magreza. Autores ${ }^{(33)}$, encontraram, em estudo realizado em Santa Catarina, quase 39,1\% de elevação dos escores z, distribuídos entre risco de sobrepeso e sobrepeso em si. Resultado praticamente igual $(37,8 \%)$, foi exposto em estudo realizado no estado de São Paulo, distribuídos da mesma maneira ${ }^{(34)}$. Os dados reforçam a informação de que os padrões nutricionais vêm se invertendo, sendo predominante, entre crianças, estados de sobrepeso e obesidade, em detrimento, de magreza ${ }^{(32)}$.

A relação $P C / l$ é uma medida de importante relevância no sentido que auxilia na mensuração crescimento do cérebro e se relaciona intrinsecamente com o adequado desenvolvimento cognitivo e motor dos lactentes ${ }^{(11)}$. Nesse aspecto, poucas alterações foram constatadas nesse parâmetro, visto que apenas um $(I, 3 \%)$ lactente estava com PC abaixo do esperado e seis lactentes (8\%) apresentaram PC acima do esperado. Em estudo recente no Ceará, as alterações apresentadas também foram pequenas nesse item, sendo mais prevalentes para acima do esperado(2). 
Em estudo realizado no Pará, considerando o desenvolvimento neuropsicomotor em que todos os domínios do TTDD-II foram avaliados, os números foram muito discrepantes em relação aos dados do presente estudo, já que $77,74 \%$ das crianças foram classificadas como suspeita de atraso( ${ }^{(18)}$. Isso pode ter acontecido devido à avaliação utilizada, não considerando os domínios separadamente. Diferentemente, no presente estudo foi utilizado apenas itens referentes ao desenvolvimento físico, incluindo avaliação motora grossa e fina.

No que se refere à avaliação de cada itemdo Teste de Denver II, estudo realizado em São Paulo com 35 crianças, o percentual de risco de atraso foi $28,6 \%$, pois também considerou separadamente os domínios cognitivo e psicossocial. Ainda, no mesmo trabalho, o percentual de itens avaliados como atraso foi de $30 \%$ e como cuidado $23,8 \%$ no DMF, enquanto nenhum item foi classificado nem como atraso, nem como cuidado no DMG ${ }^{(16)}$. Mostrando, portanto, resultados bem diferentes dos apontados na pesquisa atual.

Por fim, no que condiz à avaliação dos reflexos primitivos, em grande parte dos lactentes estavam presentes de acordo com o esperado para a idade. A avaliação dos reflexos possibilita a verificação da aptidão do lactente de se adaptar a situações ambientais e comportamentais. Dessa maneira, a ausência dos reflexos, no período que deveriam estar presentes, podem indicar atrasos na motricidade ou em outras áreas do desenvolvimento( ${ }^{(35)}$. Entretanto, no estudo não foi constatada tanta interferência, pois a avaliação do desenvolvimento motor foi satisfatória na grande maioria dos lactentes.

\section{CONCLUSÃO}

A avaliação do desenvolvimento físico envolve diferentes eixos testados além do DMF e DMG. No presente estudo, esta avaliação abrangeu avaliação das condições de saúde, imunização, antropometria, reflexos primitivos, alimentação, ambiente de cuidado, cuidadores. Para considerar o desenvolvimento infantil adequado, pode-se utilizar diferentes testes e/ou escalas. Nesse sentido, o Teste de Denver II (TTDDII) particularmente nos itens referentes ao domínio físico do desenvolvimento infantil compos a mensuração dos dados, tendo em vista sua aplicação fácil, menos onerosa e adequada para a clientela advinda na atenção primária em saúde.

Nesse sentido, no que se refere à antropometria, percebeu-se que as maiores alterações constatadas foram no IMC que revelou um perfil de lactentes acima do peso, estando em sobrepeso ou até obesos. Isso direciona a uma reflexão acerca de como os hábitos de vida atuais vêm alterando o perfil nutricional da população infantil, particularmente nas crianças já desmamadas.já em relação à avaliação de reflexos primitivos, poucas alterações importantes foram apresentadas no estudo que pudessem ser indicativas de possíveis atrasos no desenvolvimento.

No que se refere ao desenvolvimento motor fino e grosso, poucas alterações foram evidenciadas, evidenciando que as crianças estão tendo um acompanhamento adequado e são estimuladas pelos cuidadores. Entretanto, no estudo, os dados obtidos pela aplicação do TTDD-II, podem ter limitações, pela escassez de estudos que tratem de critérios para a avaliação, especificamente, do desenvolvimento motor.

Além disso, vale salientar também que, oTTDD-II não é um teste com fins diagnósticos. $O$ teste apenas alerta para possíveis alterações no desenvolvimento, sendo necessária a realização de uma avaliação com abordagem multiprofissional. No entanto, funciona como um importante método que pode contribuir para a prevenção e descoberta precoce de agravos relacionados ao desenvolvimento infantil. Por isso, torna-se importante utilizá-lo em conjunto com os parâmetros de acompanhamento do crescimento e testes de reflexos no contexto da atenção primária à saúde, no acompanhamento da criança saudável.

Portanto, neste estudo, verificou-se um preodomínio de avaliações por item e gerais (DMG e DMF) positivas entre os lactentes, o que representa um adequado desenvolvimento físico dos lactentes. Todavia, sugerem-se que novos estudos sejam realizados com aplicação do teste na íntegra com vistas ao direcionamento de ações de saúde da criança, já que o referido teste inclui as áreas física, cognitiva, pessoal-social e o fenômeno desenvolvimento integra diferentes áreas da condição biológica do ser humano. 


\section{REFERÊNCIAS}

I. Monteiro, FPM, Araujo TL, Cavalcante TF, Leandro TA, Sampaio Filho SPC. Child growth: concept analysis. Texto contexto-enferm. [Internet]. 2016 [acesso em 10 mar 2018]; 25(2):I-9. Disponível em: http://dx.doi.org/10.1590/0I0407072016003300014

2. Monteiro FPM, Joventino ES, Rouberte ESC, Rabelo PRS, Aquino MST, Silva EB. Physical development of infants: an investigation in a hospital unit. J Nurs UFPE on line [Internet]. 2017 [acesso em 10 mar 20 I8]; I I ( I ):4435-4444. Disponível em: https://periodicos.ufpe.br/revistas/revistaenfermagem/article/view/I506 I/247/6

3. Brasil. Ministério da Saúde. Secretaria de Atenção à Saúde. Departamento de Atenção Básica.

Saúde da criança : crescimento e desenvolvimento. Brasília: Ministério da Saúde, 2002. Disponível em: https://bvsms.saude. gov.br/bvs/publicacoes/crescimento_desenvolvimento.pdf

4. Brasil. Ministério da Saúde. Secretaria de Atenção à Saúde. Departamento de Atenção Básica. Saúde da criança: nutrição infantil: aleitamento materno e alimentação complementar. Brasília: Ministério da Saúde, 2009. Disponível em: http://bvsms.saude.gov.br/bvs/publicacoes/saude_crianca_ nutricao_aleitamento_alimentacao.pdf

5. Brasil. Ministério da Saúde. Secretaria de Atenção à Saúde. Departamento de Atenção Básica. Dez passos para uma alimentação saudável: guia alimentar para crianças menores de dois anos: um guia para o profissional da saúde na atenção básica. 2a.ed. Brasília: Ministério da Saúde, 2013. Disponível em: http://www.redeblh.fiocruz.br/media/I0palimsa_guia I3.pdf

6. Papalia DE, Feldman RD. Desenvolvimento humano. 12a.ed. Porto Alegre:Artmed; 2013.

7. Brasil. Ministério da Saúde. Secretaria de Atenção à Saúde. Departamento de Atenção Básica.

Saúde da criança : crescimento e desenvolvimento. Brasília: Ministério da Saúde, 2002. Disponível em: http://bvsms.saude. gov.br/bvs/publicacoes/saude_crianca_crescimento_desenvolvimento.pdf

8. Rodrigues D,Avigo EL, Leite MMV, Bussolin RA, Barela JA. Desenvolvimento motor e crescimento somático de crianças com diferentes contextos no ensino infantil. Motriz Rev Ed Fis. [Internet]. 2013 [acesso em 10 mar 20।8]; I9(3):49-56. Disponível em: http://www.scielo.br/pdf/motriz/vl9n3s0/ a08v19n3s0.pdf

9. Marques J, Sá L. Motor limitations and growth in children with cerebral palsy. J Nurs UFPE [Internet]. 2016 [acesso em 10 mar 2018]; I0(I):80-8. Disponível em: https:// periodicos.ufpe.br/revistas/revistaenfermagem/article/ view/ I0924//2209

10. Alleo LG, Souza SB, Szarfarc SC. Feeding practices in the first year of life.J Hum Growth Dev. [Internet]. 2014 [acesso em I 2 mar 2018]; 24(2): 195-200. Disponível em: http:// www.revistas.usp.br/jhgd/article/view/8I222/86108
II. Brasil. Ministério da Saúde. Secretaria de Atenção à Saúde. Departamento de Ações Programáticas Estratégicas. Coordenação Geral de Saúde da Criança e Aleitamento Materno. Caderneta de Sáude da criança. Brasília: Ministério da Saúde, 20I5. Disponível em: http://bvsms.saude.gov.br/bvs/ publicacoes/caderneta_saude_crianca_menino_l0ed.pdf

12. Pedremônico MRM, Bragatto EL, Strobilus R. Teste de triagem de desenvolvimento Denver II. Universidade Federal de São Paulo: UNIFESP, 1999.

13. Rezende MA, Beteli VC, Santos JLF. Follow-up of the child's motor abilities in day care centers and pre-schools. Rev Latino-am Enfermagem [Internet]. 2005 [acesso em 12 mar 2018]; 13 (5): 619-625. Disponível em: http://dx.doi. org/I0.1590/SOI04-II692005000500003

I4. Souza SC, Leone C, Takano OA, Moratelli HB. Desenvolvimento de pré-escolares na educação infantil em Cuiabá, Mato Grosso, Brasil. Cad Saúde Pública [Internet]. 2008 [acesso em 12 mar 2018]; 24(8): I9I7-1926. Disponível em: https://bdpi.usp.br/bitstream/handle/BDPI/I2985/art_LEONE_Desenvolvimento_de_pre-escolares_na_educacao_ infantil_em_2008.pdf?sequence= I

15. Pilz EML, Schermann LB. Determinantes biológicos e ambientais no desenvolvimento neuropsicomotor em uma amostra de crianças de Canoas/RS. Ciênc saúde coletiva [Internet]. 2007 [acesso em 12 mar 20I8]; I2(I): I8I190. Disponível em: http://dx.doi.org/I0.I590/SI4/381232007000100021

16. Moraes MW, Weber APR, Santos MCO, Almeida FA. Teste de Denver II: avaliação do desenvolvimento de crianças atendidas no ambulatório do Projeto Einstein na Comunidade de Paraisópolis. Einstein [Internet]. 2010 [acesso em 12 mar 2018]; 8(2):I49-53. Disponível em: http://dx.doi. org/10.1590/s 1679-45082010aol620

17. Brasil. Ministério da Saúde. Conselho Nacional de Saúde. Diretrizes e normas regulamentadoras de pesquisa envolvendo seres humanos. Resolução n. 466, de 12 de dezembro de 20I2. Brasília; 2012.

18. Guerreiro TBF, Cavalcante LIC, Costa EF,Valente MDR. Psychomotor development screening of children from kindergarten units of Belém, Pará, Brazil. J Hum Growth Dev. [Internet]. 2016 [acesso em 10 jul 20I8]; 26(I):I8I-I89. Disponível em: http://pepsic.bvsalud.org/pdf/rbcdh/v26n2/08.pdf

19. Pedraza DF, Santos IS. Assessment of growth monitoring in child care visits at the Family Health Strategy in two municipalities of Paraíba State, Brazil. Epidemiol. Serv. Saude [Internet]. 2017 [acesso em I3 jul 20I8]; 26(4):847855. Disponível em: http://dx.doi.org//0.5/23/s/679497420I70004000I5

20. Gomes M, Rocha CR, Henrique DM, Santos MA, Silva LR. Conhecimentos de familiares sobre os cuidados com recém-nascidos. Rev Rene [Internet]. 2015 [acesso em 01 ago 20 I8]; 16(2): 258-265. Disponível em: https://www.redalyc. org/pdf/3240/324038465016.pdf 
2I.Lima SS, Cavalcante LIC, Costa EF.Neuropsychomotor development screening of Brazilian children: a systematic review of the literature. Fisioter Pesqui. [Internet]. 2016 [acesso em 05 ago 2018]; 23(3):336-42. Disponível em: http://www. scielo.br/pdf/fp/v23n3/en_2316-91 I7-fp-23-03-00336.pdf

22. Barbieri CLA, Couto MT,Aith FMA. Culture versus the law in the decision not to vaccinate children: meanings assigned by middle-class couples in São Paulo, Brazil. Cad. Saúde Pública [Internet]. 2017 [acesso em 05 ago 20I8]; 33(2): I-II. Disponível em: http://www.scielo.br/pdf/csp/v33n2/ en_1678-4464-csp-33-02-e001733 I5.pdf

23. Ramos, CF et al. Cumprimento do calendário de vacinação de crianças em uma unidade de saúde da família. Rev Pan-Amaz Saude [Internet]. 2010 [acesso em 06 ago 20 I8]; I (2): 55-60. Disponível em: http://scielo.iec.gov.br/pdf/rpas/ $v \ln 2 / v \ln 2 \mathrm{a} 06 . p d f$

24. Boccolini CS, Boccolini PMM, Monteiro FR, Venâncio SI, Giugliani ERJ. Tendência de indicadores do aleitamento materno no Brasil em três décadas. Rev Saude Publica [Internet]. 2017 [acesso em 06 ago 20 I8]; 5 I (108): I-9. Disponível em: http://www.revistas.usp.br/rsp/article/view/ / 40946

25. Souza ES, Magalhães LC. Motor and functional development in infants born preterm and full term: influence of biological and environmental risk factors. Rev Paul Pediatr [Internet]. 2012 [acesso em 06 ago 2018]; 30(4): 462-70. Disponível em: https://doi.org/I0.1590/S0 I03-058220I2000400002

26. Nunes JT, et al. Quality of prenatal care in Brazil: review of published papers from 2005 to 2015. Cad. Saúde Colet [Internet]. 2016 [acesso em 06 ago 2018]; 24(2): 25226I. Disponível em: http://www.scielo.br/pdf/cadsc/v24n2/ |4|4-462X-cadsc-24-2-252.pdf

27. Aquino LA. Acompanhamento do crescimento normal. Revista de Pediatria SOPERJ [Internet]. 20I I [acesso em 07 ago 2018]; 12 (I): I5-20. Disponível em: http://revistadepediatriasoperj.org.br/detalhe_artigo.asp?id=553

28. Renz BM, Cunha KA, Gehm LL, Souza MA, Renner FW. Prevalência de recém-nascidos pequenos para idade gestacional e fatores associados. Bol Cient Pediatr [Internet]. 2015 [acesso em 07 ago 2018];4(I): I7-2I.Disponível em: https:// www.sprs.com.br/sprs20 I3/bancoimg/I509/522। I55bcped_v4_nl_a5.pdf

29. Pereira JF, Formiga CKMR, Vieira MEB, Linhares MBM. Influência dos fatores biológicos e socioeconômicos no desenvolvimento neuropsicomotor de pré-escolares. Revista Saúde e Pesquisa [Internet]. 2017 [acesso em 07 ago 2018]; I0(I): 135-I44. Disponível em: https://periodicos.unicesumar.edu.br/index.php/saudpesq/article/ view/5788

30. Silva EM, Miranda CT, Puccini RF, Nóbrega FJ. Day care centres as an institution for health promotion among needy children: an analytical study in São Paulo, Brazil. Public Health [Internet]. 2000 [acesso em 07 ago 2018]; II4(I): 385-388. Disponível em: https://www.ncbi.nlm.nih.gov/pubmed/ I I03546 I

3I. Rodrigues VC, Mendes BD, Gozzi ASF, Santana RG, Matioli G. Deficiência de ferro, prevalência de anemia e fatores associados em crianças de creches públicas do oeste do Paraná, Brasil. Rev Nutr [Internet]. 201 I [acesso em 08 ago 2018]; 24(3): 407-420. Disponível em: https://doi. org/I0.1590/SI4I5-52732011000300004

32. Ezzati M.Worldwide trends in body-mass index, underweight, overweight, and obesity from 1975 to 2016: a pooled analysis of 2416 population-based measurement studies in 128.9 million children, adolescents, and adults. The Lancet [Internet]. 2017 [acesso em 08 ago 2018]; 390(I0II3): 2627-2642. Disponível em: https://www.ncbi.nlm.nih.gov/ pubmed/29029897

33. Dallabona A, Cabral SC, Höfelman DA. Variáveis infantis e maternas associadas à presença de sobrepeso em crianças de creches. Rev Paul Pediatr [Internet]. 2010 [acesso em 08 ago 2018]; 28(4): 304-3I3. Disponível em: https://doi. org/10.1590/S0103-05822010000400004

34. Nascimento VG, Silva JP, Bertoli CJ, Abreu LC, Valenti VE, Leone C. Prevalência de sobrepeso em crianças pré-escolares em creches públicas:um estudo transversal. Med J [Internet]. 2012 [acesso em 08 ago 2018]; I 30(4): 225-229. Disponível em: https://doi.org/I0.I590/SI516-31802012000400004

35. Grave MTQ, Sartori V. Avaliação de crianças nascidas prétermo a partir dos reflexos neonatais, frequência respiratória e doenças associadas. Caderno pedagógico [Internet]. 2012 [acesso em 08 ago 20I8]; 9(2):|39-I5I. Disponível em: http://www.univates.br/revistas/index.php/cadped/article/view/862.

Recebido: 2020-04-2I Aceito: 2020-06-0 I 